\title{
Training structure of powerlifters with regard to biological rhythms and operational functional condition
}

\author{
Shutova T.N. ${ }^{\mathrm{ABCDE}}$, Vysotskaya T.P. ${ }^{\mathrm{BD}}$ \\ Plekhanov Russian University of Economics, Russia
}

Authors' Contribution: A - Study design; B - Data collection; C - Statistical analysis; D - Manuscript Preparation; E - Funds Collection.

\begin{abstract}
Purpose: $\quad$ to develop and experimentally confirm the structure of training cycles, training load and sports results with regard to the biological rhythms and functional condition of powerlifters.

Material: $\quad$ the study involved qualified powerlifters $(n=40)$. The diagnostic procedure was performed on the Omega hardware complex. Ostberg questionnaire was applied to determine the athlete's chronotype. According to the results of the questionnaire, athletes were divided into morning, evening and mixed chronotypes. In the experimental group ( $\mathrm{N} 1, \mathrm{n}=20$ ), intensive training was conducted at the peak of the biological rhythm. Medium and low-intensity training was performed in the phase of reducing the biorhythms activity. Standard training was performed in the control group $(\mathrm{N} 2, \mathrm{n}=20)$, without regard to chronotypes.

Results: $\quad$ it was determined that the application of non-standard means of athletes training at the peak of the biological rhythms activity and in the rising phase of the biogram significantly influences on the increase in maximum strength results. Non-standard means include Crossfit training and a reverse pyramid (the maximum weight is applied at the beginning of the training, in the subsequent approaches the weight reduces, the number of repetitions increases). It is determined the significant differences between groups in the control exercises: hanging pull-up/chin up, parallel bar dips, hanging on the crossbar.

Conclusions: the training of qualified powerlifters should be based on their chronotype. The main mean of the training process optimizing of athletes should be a biogram, reflecting the physical, emotional and intellectual biological rhythm.

Keywords: chronotype, planning, cycle, training, competition.
\end{abstract}

\section{Introduction}

The dynamic development of powerlifting draws the attention of scientists to the development of the qualified athletes training content and the methodological bases of training cycles structure $[1,2]$. In recent years, there is a sufficient number of scientific works devoted to powerlifters training at the primary level. It is determined the optimal combinations of means, methods, and parameters of power and speed-power training load. It was revealed the effect of power training on the athletes' health $[3,4]$. The researchers confirm that athletes' training should be based on individual training programs. This allows you to increase the level of their special power fitness and effectiveness of performances in competitions $[5,6]$.

The effective variants of training load structure were revealed in works of Kholopov and Rybalsky. Load in the weekly and monthly cycles allow optimizing the athletes' training $[7,8]$. It was developed and put into practice the methodical recommendations of pre-competitive weight regulation by powerlifters. Recommendations are based on identifying the optimal was of weight reduction and its effect on athletic performance [9]. Recovery processes in power triathlon are revealed in the studies of Khitrov, Stetsenko, Artyushenko [10, 11]. The research by Dalsky confirmed the effective application of the powerlifter' functional index. The operational control and training load correcting are made on the index base [12].

However, these works do not consider the issue (c) Shutova T.N., Vysotskaya T.P., 2019

doi:10.15561/20755279.2019.0107 of training structure based on a biogram. There are not enough scientific works in powerlifting based on the daily, weekly, monthly activity of biological rhythms. Not enough hardware methods to control the functional status of athletes [13]. Increasing requirements for the implementation of maximum strength abilities, the level of reliability of training of athletes led to the search for new ways of sports training [14]. Depending on the athlete's biological rhythm, the voltage level of the body's systems is changing. The voltage of functional systems is essential for achieving high results of powerlifters [15, 16]. Differences between the maximum and minimum sports results during the day are $10-25 \%$. Therefore, training sessions in the morning and evening hours are justified, depending on the biological rhythm. Such an approach can be a reserve for improving the athletic performance of qualified athletes in the annual training cycle. Shaposhnikova, Taymazov found that $52.3 \%$ of sports injuries were received on critical days of biorhythms. And in the positive phase of biorhythms, athletes achieve better results than in the negative phase $[17,18]$. Therefore, studies on the management of the training process based on the activity of biological rhythms, planning training time depending on the chronotype are relevant.

The purpose of the study: to develop and experimentally justify the construction of training cycles, training load, and athletic performance, taking into account the biological rhythms and functional state of qualified powerlifters. 


\section{Material and methods}

The participants. The experimental group $(\mathrm{N} 1, \mathrm{n}=$ 20) consisted of powerlifters 18-25 years old with the following qualifications: 7 - candidates for master of sports, 4 - masters of sports, 9 - I category; the control group had almost identical qualifications $(\mathrm{N} 2, \mathrm{n}=20)$.

Design of the study. The experiment was conducted on the basis of the Tchaikovsky State Institute of Physical Culture (Tchaikovsky, Russia). The N1 group trained 4 times per week, 2.5 hours long. Group N2 trained 3 times per week: 2 times per week 3 hours long and 1 time 2 hours long.

Improving the training process of qualified powerlifters was to distribute them according to the biological rhythm. To solve this problem, athletes have passed diagnostics on the Omega hardware complex (1st stage). It was detected hours of biological activity during the day, week, month. The control included indicators of the level of adaptive abilities, psycho-emotional state, fitness level, energy supply, the tension of regulatory systems. The express technique "Omega" characterized the integral indicator of the functional state and sports form of each of the athletes. The technique has defined the "zones" of the functional state: "Red" - overstrain, overwork, the significant decrease in the functional state. The "green" zone is an optimal functional state, a high level of adaptation and fitness. The "yellow" zone is a borderline state, a decrease in reserves of the functional state, a violation of adaptive capabilities. The Omega complex took into account: the internal and external components of biological rhythms; adaptation processes after high-intensity training sessions; recovery time.

The next step in determining the chronotype was Ostberg questionnaire (stage 2) [19]. According to the results of the questionnaire, athletes were divided into morning, evening and mixed chronotypes. The questionnaire can be attributed to the subjective and objective means of determining biological rhythms. In conclusion, the questionnaire was applied in the form of interviewing (stage 3). It was conducted to determine the subjective components of the psychophysical condition, adaptation to physical loads, and general condition during and after training. The individual biogram (14-30 days) for every athlete is distributed over periods of 4-8 days. The structure of training effects was chosen depending on the phase of biological rhythms activity (Fig. 1). The recovering training load was applied in minimal biorhythm activity (phase 1 , means from $-80,-100$, to -80 points - the lowest point of the graph). During this period, the recommended physical load was $40-60 \%$ of the maximum weight. The support training was performed in the ascending activity (phase 2 , from $-80,-50$, to 50 points - the average ascending part of the graph),. The loads 60$75 \%$ of the maximum weight are realized in these days. The intensive training was conducted with a maximum load of $75-105 \%$ and higher at the peak of biorhythm activity (phase 3 , from 50 to 100 and again 50 points the top point of the graph). The training process included $50-75 \%$ of the maximum load in descending activity (phase 4, from 50, 0 points and up to -80 points - the average descending part of the graph). Biogram reflects the physical, emotional, intellectual biological rhythm of the athlete (Fig. 1). The plans of training loads were developed for every phase of biorhythms activity.

\section{Statistical analysis}

Statistical data processing was carried out by the method of variation statistics with the calculation of the arithmetic mean value, standard deviation, and verification of the results of the study for the significance of differences at the 5\% significance level [20]. Student's $\mathrm{t}$-criterion was applied in the evaluation of significant differences. Mathematical processing was carried out applying the Excel 2010 tabular processor and the StatPlus2009 program.

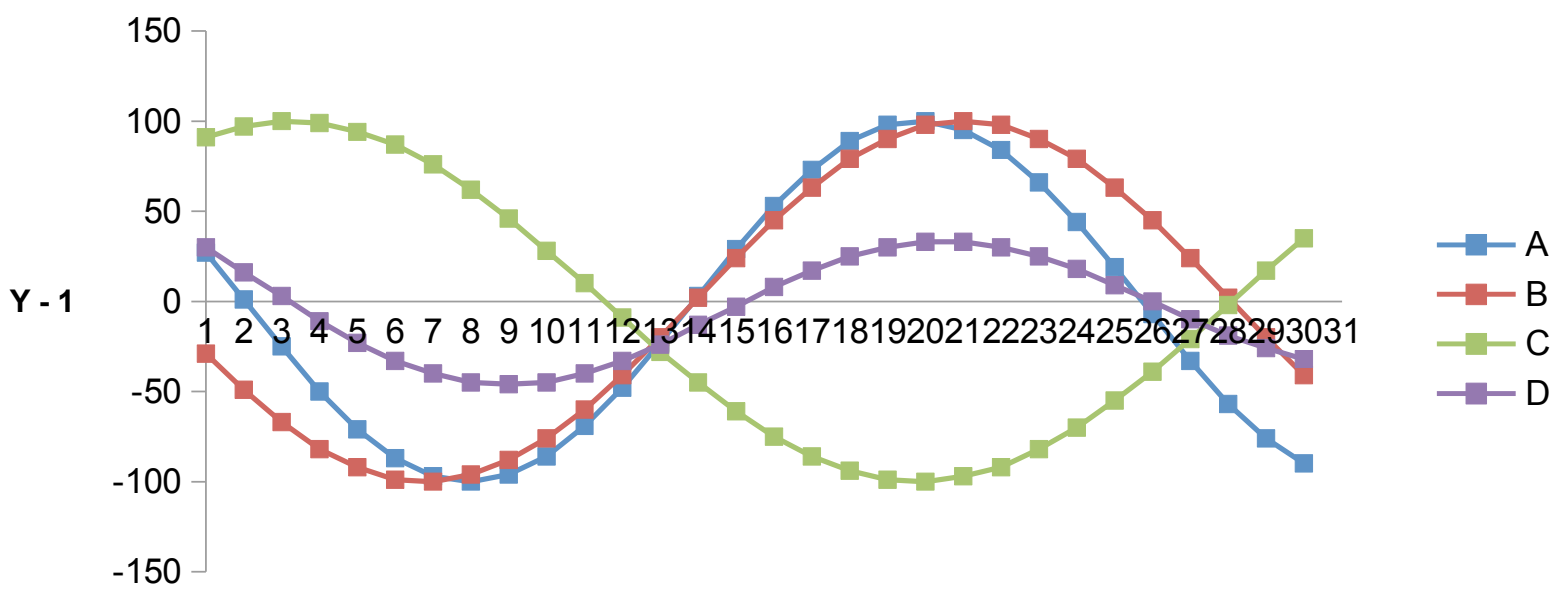

$x-2$

Y-1 - Points; , X-2 - Days; A - Physical biorhythm; B - Emotional biorhythm; C - Intellectual biorhythm; D General state.

Fig. 1. Individual biorhythm graph for 30 days (point) 


\section{Results}

In the experiment were obtained data concerning the types of training load for qualified powerlifters (limiting, supporting, recovering), their combination in the structure of the occupation and training cycles. The experiment included non-standard means of training: Crossfit training and the inverse pyramid (the maximum weight is applied at the beginning of the training, in subsequent approaches the weight reduces, the number of repetitions increases).

The construction of the training process considering the biological rhythms and functional status has significantly improved the athletic performance. In the N1 group, the results are higher compared to the $\mathrm{N} 2$ group. The squats were $197.9 \mathrm{~kg}$ (group N1) versus $184.7 \mathrm{~kg}$ (group N2). The bench press was $147 \mathrm{~kg}$ (group N1) versus $129 \mathrm{~kg}$ (group $\mathrm{N} 2$ ). The dynamics of the deadlift reached $187 \mathrm{~kg}$ (group N1) in comparison with $175 \mathrm{~kg}$ (group N2). The sum of three control exercises was $532.2 \mathrm{~kg}$ (group N1) and 489.6 $\mathrm{kg}$ (group N2) (Table 1). It is proved the effectiveness of training influences on the basis of special physical fitness of athletes in the control exercises: hanging pull-up/chin up, parallel bar dips, hanging on the crossbar.

The effectiveness of experimental training of powerlifters is proved by the optimal functional condition of athletes of the $\mathrm{N} 1$ group, in comparison to the $\mathrm{N} 2$ group. The total value of the functional condition ("Omega" method) is: $85.7 \%$ (group N1), $71.9 \%$ (group N2) (maximum 100\%). Adaptation to physical loads is: $84.3 \%$ (group N1) and 71.4\% (group N2). The level of energy supply is $81.9 \%$ (group N1) and $71.6 \%$ (group N2). The level of fitness is $93 \%$ (group N1), $80.2 \%$ (group N2). The psycho-emotional condition is: $80.8 \%$ (group N1), $71.0 \%$ (group N2) (maximum 100\%). The level of adaptation and reserve capabilities of the cardiovascular and respiratory systems is 7.3 c.u. (group N1), 10.1 c.u. (group N2). Vital capacity of the lungs does not have significant differences in results. The initial and final values are practically the same and are 4000-4300 ml. (group N1), 4100-4200 ml. (group N2)

Blood pressure does not have significant differences. The revealed values of systolic and diastolic pressure at rest correspond to the parameters of the norm. Conducted training are not contributed to the increase in blood pressure.

\section{Discussion}

The objectification of the training process management of qualified powerlifters remains relevant. It requires the development of integral criteria, models for evaluating the physical, functional and psychological components. It is also necessary to consider biological rhythms, the development of the most rational options for training load structure. Attention should be paid to the fact that a significant part of the research is devoted to determining the optimal volumes and intensity of training load. Rybalsky developed a classification of the volume and intensity of training load. The classification gives an objective evaluation of the magnitude and direction of training effects. In the micro cycles of the basic stage of preparation, the volume of exercises should be squats $45 \%$; bench press $-35 \%$; deadlift $-20 \%$. The volume of the exercises should be $14-20 \%$ - high intensity; $41-44 \%$ - medium intensity; 39-42\% - low intensity [8]. In our study, the volume and intensity of training influences are determined by the phase of activity of biological rhythms during the month; daily activity; operational functional

Table 1. Results of the main power indicators and special physical fitness in the experiment

\begin{tabular}{|c|c|c|c|c|}
\hline Exercises & Group & $2015(X \pm \sigma)$ & $2016(X \pm \sigma)$ & $2017(X \pm \sigma)$ \\
\hline \multirow{2}{*}{ 1. Barbell Squat (kg) } & N1 & $167.6 \pm 19.5$ & $183.5 \pm 15.7$ & $197.9 \pm 16.5^{*}$ \\
\hline & N2 & $170.9 \pm 10.5$ & $176.7 \pm 16.3$ & $184.7 \pm 9.2 *$ \\
\hline \multirow{2}{*}{ 2. Bench Press With Barbell (kg) } & N1 & $111.7 \pm 10.5$ & $125.3 \pm 8.7$ & $147.1 \pm 20.4^{* *}$ \\
\hline & N2 & $109.3 \pm 9.1$ & $119.5 \pm 6.3$ & $129.7 \pm 8.7 *$ \\
\hline \multirow{2}{*}{ 3. Barbell Deadlift (kg) } & N1 & $163.7 \pm 14.1$ & $182.5 \pm 13.3^{*}$ & $187.2 \pm 9.6 * *$ \\
\hline & N2 & $165.2 \pm 12.4$ & $170.6 \pm 11.5$ & $175.2 \pm 10.4$ \\
\hline \multirow{2}{*}{$\begin{array}{l}\text { 4. The sum of three control } \\
\text { exercises }(\mathrm{kg})\end{array}$} & N1 & $443 \pm 21.9$ & $491.3 \pm 18.6^{*}$ & $532.2 \pm 23.7^{* *}$ \\
\hline & N2 & $445.4 \pm 24.9$ & $466.8 \pm 12$ & $489.6 \pm 20.7^{*}$ \\
\hline \multirow{2}{*}{ 5. Handgrip Strength Test (right), kg } & N1 & $44.8 \pm 5.3$ & $46.6 \pm 5.3$ & $46.9 \pm 4.7$ \\
\hline & N2 & $46.3 \pm 5.8$ & $46.6 \pm 5.2$ & $48.8 \pm 5.1$ \\
\hline \multirow{2}{*}{ 6. Handgrip Strength Test (left), kg } & N1 & $42.1 \pm 5.6$ & $43.6 \pm 5.7$ & $44.2 \pm 5.4$ \\
\hline & N2 & $45.7 \pm 5.6$ & $45.6 \pm 5.5$ & $46.8 \pm 5.2$ \\
\hline \multirow{2}{*}{$\begin{array}{l}\text { 7. Pull-Up / Chin Up Test (upper } \\
\text { crossbar), quantity of times }\end{array}$} & N1 & $16.5 \pm 4.8$ & $18.9 \pm 5.1$ & $27.6 \pm 3.9 * *$ \\
\hline & N2 & $15.9 \pm 4.4$ & $16.3 \pm 3.9$ & $20.1 \pm 3.5^{*}$ \\
\hline \multirow{2}{*}{$\begin{array}{l}\text { 8. Parallel bar dips (quantity of } \\
\text { times) }\end{array}$} & N1 & $18 \pm 5.3$ & $37.8 \pm 8.7 * *$ & $38.3 \pm 5.5^{* *}$ \\
\hline & N2 & $22.7 \pm 7.8$ & $24.6 \pm 7.8^{*}$ & $25.6 \pm 6.2^{*}$ \\
\hline \multirow{2}{*}{$\begin{array}{l}\text { 9. Bent Arm Hang Test (two hands), } \\
\text { sec }\end{array}$} & N1 & $38.5 \pm 10.9$ & $45.5 \pm 13.4$ & $46.1 \pm 12.8$ \\
\hline & $\mathrm{N} 2$ & $38.2 \pm 10.7$ & $39.7 \pm 10.6$ & $41.1 \pm 9.7$ \\
\hline
\end{tabular}

Note: $\mathrm{X}$ - arithmetic mean, $\sigma$ - standard deviation; EG - experimental group, CG - control group; * significance of differences $(p<0.05), * *$ - intergroup significance of differences $(p<0.05)$. 
condition.

The structure of the training means of qualified powerlifters includes special strength training $(74.4 \%$ of the time a year); general physical training (18.7\%); pedagogical means of recovery $(3.7 \%)$. The intensity dynamics by the number of barbell lifts is in the range of $70-112 \%$ of the individual maximum [21]. In our study, the intensity of the barbell lifts is in the range of $40-105 \%$ of the individual maximum. It depends on the phases of biorhythms.

Experimental application of static power stresses in the study allowed an increase in the initial strength indices of the arms (by 35.4\%), leg muscles (by 33.4\%), back muscles (by 36.7\%) [22]. Vorozheikin identified the rank structure of the factors that determine the high level of power fitness of powerlifters. They include: individual strength abilities (19.7\%); high level of motivation to achieve a good result in competitions - 17.8\%; high degree of concentration (15.5\%); good moral-volitional fitness $(14.3 \%)$; correspondence of the volume and intensity of the load to individual peculiarities (10.7\%); accuracy of performance depending on anatomical and morphological features $(9.5 \%)$; high relative load during the training process $(7.2 \%)$; the ability to quickly recover in the process of strength training (5.3\%) [6].

Analysis of other studies has shown that the emotional rhythm lasts 28 days. It is associated with changes in mood, the reactivity of the body. The intellectual rhythm lasts 33 days. It is associated with mental performance. Many athletes are dominated by weekly and two-week biorhythms. Biorhythms are determined by the indices of the minute respiratory volume, heart rate, temperature and body mass, energy metabolism [23]. Our study showed that considering the biorhythms of athletes it is possible: to develop maximum strength capabilities; expect intense training at the peak of the biological rhythm. The medium

\section{References}

1. Verkhoshansky Yu. The principles of the organization of high-class athletes training in the annual cycle. Theory and Practice of Physical Culture, 2006;2:24-31.

2. Dodonov AP, Shutova TN. Training of qualified powerlifters on the basis of biorhythmological approach. Theory and Practice of Physical Culture, 2013;10:20.

3. Perov PV. The content of physical training at the initial stage of powerlifting [dissertation]. St. Petersburg; 2005. (in Russian)

4. Ostapenko LA. Features of the training process in the power triathlon at the stage of selection and initial training [dissertation]. Moscow; 2002. (in Russian)

5. Kostryukov VV. Improvement of special power training of qualified powerlifters on the basis of application of exercises with variable weights [dissertation]. Cheboksary; 2011. (in Russian)

6. Vorozheykin OV. Power training of powerlifters of various sports qualification on the basis of individual training programs [dissertation]. Saint-Petersburg; 2010. (in Russian)

7. Kholopov VA. Construction and maintenance of training loads in the annual cycle of training of powerlifters of the senior categories [dissertation]. Moscow; 2008. (in Russian) and low-intensity load should be applied in the biogram reduction phase. Therefore, the structure of the training load for 14-30 days (biogram) is justified.

The results of our research allow us to evaluate objectively the planning of preparation cycles; evaluate training load and sports results. It is necessary to consider the biological rhythms and operational functional status of athletes (the method of "Omega). The results of our study are in good agreement with the data of other authors $[15,24]$. Training athletes considering the phases of biorhythms (4 phases for 4-7 days) demonstrates a significant increase in athletic indicators. The study allows to characterize the possibilities of practical application of the system of tools and methods for individualizing the training process of athletes.

\section{Conclusions}

The training of qualified powerlifters should include 4 training per week 2,5 hours long. Training should be conducted: in the morning $(8.00-10.00$, for the morning type of activity of biological rhythms); in the evening (18.00-20.00, for the evening type of activity of biological rhythms). Training (morning or evening) should be conducted for a mixed type of activity. The main means of athletes training process optimizing should be a biogram for 14 and 30 days. Biogram reflects the physical, emotional, intellectual biological rhythm of the individual. To increase the maximum strength results of athletes, it is recommended to apply non-standard means of training. Non-standard means include Crossfit workouts and a reverse pyramid. These means must be applied at the peak of the activity of biological rhythms and in the ascending phase of the biogram.

\section{Conflict of interest}

The authors declare that there is no conflict of interest.
8. Rybalsky P I. Structure and content of training microcycles of different orientation depending on the characteristics of competitive exercises in powerlifting [dissertation]. Moscow; 2000. (in Russian)

9. Perfileva EV. Pre-competition regulation of body weight of powerlifters of high qualification [dissertation]. Malakhovka; 2010. (in Russian)

10.Khitrov MV. Method of restoration of the musculoskeletal system of qualified athletes in the power triathlon [dissertation]. Tula; 2013. (in Russian)

11. Stetsenko AI, Artyushenko AF. Adaptation to physical loads development of sports form in powerlifting. International scientific methodic conference "Physical culture, sport and health of the nation", Vinnitsa; 1994. p. 384-386. (in Russian)

12.Dalsky DD. Correction of training load in powerlifting on the basis of operational control methods [dissertation]. SaintPetersburg; 2013. (in Russian)

13.Zareian E, Rabbani V, Saeedi F. The Effect of Physical Biorhythm Cycle on some Physical Fitness Factors of Adolescent Volleyball Players. Annals of Applied Sport Science, 2014;2(1):11-20. doi: 10.18869 / acadpub. aassjournal.2.1.11 
14.Shutova TN, Dodonov AP. Modeling of training process of skilled powerlifters. Moscow; 2017. (in Russian)

15.Maura S. Bio-periodicity and its influence on the football results obtained by the student teams during the "university spring" sport competition. Journal of Physical Education and Sport, 2009;4:115-118.

16.Moldovan E, Enoiu RS, Ruxanda RA, Leibovici A. The influence of the human biorhythm in the performance sport activity. Gymnasium, 2011;12:34-46.

17.Shaposhnikova VI. Biorhythms-health hours. Moscow: Soviet sport; 1991. (in Russian)

18.Shaposhnikova VI, Narcissusov RP, Barbaras NA. Longterm and annual human cycles.2nd ed. Moscow: Triad X; 2000. (in Russian)

19.Stepanova SI. Biorhythmological aspects of the problem of adaptation. Moscow: Nauka; 1986. (in Russian)

20.Ivanov VS. Fundamentals of mathematical statistics. Moscow: Physical education and sport; 1990. (in Russian)

21.Trishin ES. Special training of qualified powerlifters in weekly microcycles of the preparatory period [dissertation], Moscow; 2011. (in Russian)

22.Zverev VD. Operational control of the functional state of powerlifters. Scientific Notes of the University $P F \quad$ Lesgaft, 2012;5:42-46. (in Russian) https://doi.org/10.5930/issn.1994-4683.2012.05.87.p42-46

23.Shaposhnikova VI, Taymazov VA. Chronobiology and sports. Moscow, 2005. (in Russian)

24.Raut TS, Kaware SHH. Comparative study of Biorhythms with Various physical fitness components. International Referred Research Journal, 2011;4:32-33.

25.Aksenov MO, Aksenova OV. Construction of training process of athletes of weightlifting sports taking into account the data of bioimpedance analysis. Theory and Practice of Physical Culture, 2015;12:74-76.

26.Antoniuk O, Pavlyuk Y, Vynogradsky B, Pavlyuk O, Chopyk T, Soltyk O. Improvement of technical preparedness of elite female weightlifters with different types of body build. Journal of Physical Education and Sport, 2017;17(4):2428-2436. https://doi.org/10.7752/jpes.2017.04270

27.Castillo F, Valverde T, Morales A, Perez-Guerra A, GarciaManso J. Maximum power, optimal load and optimal power spectrum for power training in upper-body (bench press): a review. Rev Andal Med Deporte, 2011;5(1):18-27. https://doi.org/10.1016/S1888-7546(12)70005-9

28.Chaouachi M, Chaouachi A, Chamari K., Chtara M, Feki Y, Amri M, Trudeau F. Effects of dominant somatotype on aerobic capacity trainability. $\mathrm{Br} J$ Sports $\mathrm{Med}$, 2005;39:954-959. https://doi.org/10.1136/bjsm.2005.019943

29.Dodonov AP, Shutova TN. Operational functional state in the preparation of qualified powerlifters. Theory and Practice of Physical Culture, 2015;3:26.

30.Dehghan AM. The psychological factors affecting investment in stock. A Monthly Magazine TADBIR on Management, 2008;196: 60-63.

31.Ezanloo B, Ebrahimi S, Ghavam Habibi M, Asgarabad. The Investigation of the Validity of Intellectual Cycles of Biorhythm through Cognitive Functions. Research in Psychological Health, 2007;1(3):38-50.

32.Fister I, RauterS, Yang XS. Planning the sports training sessions with the bat algorithm. Neurocomputing, 2015;14:993-1002. https://doi.org/10.1016/j.neucom.2014.07.034

33.Guz SM. The structure and content of the macrocycles on the stage of advanced training in powerlifting. Scientific Notes University of PF Lesgaft, 2011;8:61- 66. (in Russian)

34.Gutnik B, Zuoza A, Zuoziene I, Alekrinskis A, Nash D, Scherbina S. Body physique and dominant somatotype in elite and low-profile athletes with different specializations. Medicina, 2015;51: 247-252. https://doi.org/10.1016/j.medici.2015.07.003

35.Hoseini SM, Mehdizadeh A, Ashrafi. Biorhythm and Educational Performance: (Students of Islamic Azad University, Firoozkuh Branch. Pajouheshgar). Journal of Management, 2009;6(13):70-6.

36.Helms E, Fitschen PJ, Aragon A, Cronin J, Shoenfeld BJ. Recommendations for natural bodybuilding contest preparation: Resistance and Cardiovascular Training. The Journal of Sports Medicine and Physical Fitness, 2014;1:37.

37.Hale BD, Roth AD, DeLong RE, Briggs MS. Exercise dependenceand the drive formuscularity in male bodybuilders, power lifters, and fitness lifters. Body Image, 2010;7(3):234-9. https://doi.org/10.1016/j.bodyim.2010.02.001

38.Imaran M, Hussian I, Murtaza S, Jabin F, Ashad Bari M. A comparative study of bodybuilders and weightlifters on somatotypes. Journal of Education and Practice, 2015;3: online

39.Jensen AM. A mind-body approach for precompetitive anxiety in power-lifters: 2 case studies. Journal of Chiropractic Medicine, 2010;9(4): 184-92. https://doi.org/10.1016/j.jcm.2010.08.003

40.Kostyunina LI, Ilkin AN, Birjevay AO. Exchange planning of training loads of qualified powerlifters in the preparatory period of the annual cycle. Volga Region Pedagogical Search, 2017; 4 (22):133-138.

41.Korkmaz S, Harbili E. Biomechanical analysis of the snatch technique in junior elite female weightlifters. Journal of Sports Sciences, 2016;34(11):1088-93. https://doi.org/10.1080/02640414.2015.1088661

42.Kryazhev VD. Methodology of development, the preservation and restoration of motor capabilities in athletic training and Wellness physical education. [dissertation]. Moscow; 2003. (in Russian)

43.Keogh JWL, Hume PA, Pearson SN, Mellow P. Anthropometric dimensions of male powerlifters of varying body mass. Journal of Sports Sciences, 2007;25(12): 1365-76. https://doi.org/10.1080/02640410601059630

44. Shutova TN. Fitness and computer technologies in physical education of students. Theory and practice of physical culture, 2016;3:101.

45.Shutova T. N. Modeling of fitness technologies in the process of physical education of students on the basis of diagnostics "ESTECK system COMPLEX". Theory and practice of physical culture, 2017;9:30-32.

46. Silva A, Mendes D, Oliveira E, Almeida H, Ascenso R. Bodyshifter-softwaretodetermineandoptimizeanindividual's somatotype. Procedia Technology, 2014;16: 1456-1461. https://doi.org/10.1016/j.protcy.2014.10.165 


\section{Information about the authors:}

Shutova T.N.; http://orcid.org/0000-0002-6249-0944; tany-156@rambler.ru; Plekhanov Russian University of Economics; Stremyanny lane 36, Moscow, 117997, Russian Federation.;

Vysotskaya T.P. (Corresponding Author); http://orcid.org/0000-0002-2430-0345; golubnichaya2010@yandex.ru; Plekhanov Russian University of Economics; Stremyanny lane 36, Moscow, 117997, Russian Federation.

\section{Cite this article as:}

Shutova TN., Vysotskaya TP. Training structure of powerlifters with regard to biological rhythms and operational functional condition. Physical education of students, 2019;23(1):45-50.

https://doi.org/10.15561/20755279.2019.0107

The electronic version of this article is the complete one and can be found online at: https://sportedu.org.ua/index.php/PES/issue/archive

This is an Open Access article distributed under the terms of the Creative Commons Attribution License, which permits unrestricted use, distribution, and reproduction in any medium, provided the original work is properly cited http://creativecommons.org/licenses/by/4.0/deed.en

Received: 30.01 .2019

Accepted: 19.02.2019; Published: 28.02.2019 